\title{
Assessing ovulation detection performance in commercial dairy herds using progesterone concentrations from limited numbers of strategically collected milk samples
}

\author{
J. M. Morton ${ }^{* 1,2}$ and P. C. Wynnt ${ }^{3}$ \\ ${ }^{*}$ Faculty of Veterinary Science, The University of Melbourne, 250 Princes Highway, Werribee, Victoria 3030 Australia and School of Veterinary \\ Science, The University of Queensland, Queensland 4072 Australia \\ †Dairy Research Unit, Faculty of Veterinary Science, The University of Sydney, PMB 3, Camden, New South Wales 2570 Australia
}

\begin{abstract}
It is important to assess ovulation detection performance in commercial dairy herds both to investigate low reproductive performance and to enable herd managers to monitor the effectiveness of their system for detecting ovulations. A method was developed to assess ovulation detection performance that uses limited numbers of strategically collected milk samples, assesses performance over the period when herd managers are making maximal effort to detect ovulations, and when assessing proportions of ovulations detected, accounts for false positive diagnoses of estrus and for cows that have not recommenced postpartum ovulatory cycles. Milk was sampled from cows not diagnosed in estrus early in the breeding program (about d 26 in year-round calving herds and d 22 in seasonal calving herds); milk samples were also collected from cows on the day of insemination. Cows with high milk progesterone concentrations were assumed to have had undetected ovulations and false positive diagnoses of estrus, respectively. The method was successfully implemented in 161 of 167 commercial dairy herds. Positive predictive values (PPV; the proportions of ovulation diagnoses where ovulation was, in fact, imminent) were generally high in both year-round and seasonal calving herds (median values were 0.96 and 0.97 , respectively), but $25 \%$ of herds had PPV $<0.95$. Ovulation detection sensitivities (ODS) were low in most year-round calving herds, but many seasonal calving herds had high ODS values; median ODS were 0.73 and 0.94 , respectively. However, in $25 \%$ of seasonal calving herds, ODS was $<0.91$. These findings indicate that this method for assessing ovulation detection performance can be successfully implemented in
\end{abstract}

Received November 23, 2009.

Accepted April 6, 2010.

${ }^{1}$ Corresponding author: john.morton@optusnet.com.au

${ }^{2}$ Current address: PO Box 2277, Geelong, Victoria 3220 Australia.

${ }^{3}$ Current address: School of Animal and Veterinary Sciences, Charles Sturt University, Locked Bag 588, Wagga Wagga, New South Wales 2678 Australia. commercial dairy herds with appropriate professional support. The wide range of ODS and the absence of correlation between ODS and PPV suggest that it is possible for managers of many commercial herds in Australia to achieve increased reproductive efficiency through increases in ODS and PPV.

Key words: estrus, ovulation, detection, dairy

\section{INTRODUCTION}

In dairy herds where AI is used, management strategies are necessary to identify cows in which ovulation is imminent. Most strategies are based on detection of behavioral changes exhibited by cows during estrus and the success is reflected as estrous detection performance for the herd. Rather than aiming to detect estrus, herd managers are primarily aiming to identify cows in which ovulation is imminent. Although this most commonly relies on detection of estrus, cows can ovulate without being mounted (Shipka, 2000) and perhaps without showing other behavioral signs usually associated with estrus. Some methods for detecting ovulation do not rely on behavioral signs of estrus; these include methods that use body temperature (Geers et al., 1997), vaginal mucus conductivity (Meena et al., 2003), and in-line milk progesterone sensors (Delwiche et al., 2001).

There are 2 ovulation detection errors: false positive diagnoses (imminent ovulation diagnosed in cows where ovulation is not imminent) and undetected ovulations. Inseminations following false positive diagnoses reduce the overall proportion of AI that result in conception (Gowan et al., 1982). Undetected ovulations during the breeding period delay conception, and a high frequency of undetected ovulations reduces primary measures of herd reproductive performance such as mean calving to conception interval (Gaines et al., 1993).

It is important to assess ovulation detection performance in commercial dairy herds when investigating low herd reproductive performance and to enable herd managers to monitor the effectiveness of their system for detecting ovulations. This can be assessed using 2 
indices: positive predictive value (PPV) for ovulation diagnoses and ovulation detection sensitivity (ODS). The PPV for ovulation diagnoses (also referred to as detection "accuracy") is the proportion of diagnoses of imminent ovulation where ovulation is imminent. This is equal to 1 minus the proportion of diagnoses that are false positives. Positive predictive values are usually assessed for ovulation diagnoses that are followed by AI; in research studies, this has been estimated based on milk progesterone concentration on day of $\mathrm{AI}$ in a representative sample (Gowan et al., 1982; Cavestany and Galina, 2001a). Cows with a functional corpus luteum at the time of AI have high progesterone concentrations. Repeated ultrasonic examination of ovaries has also been used to estimate PPV (Gümen et al., 2003).

Ovulation detection sensitivity is the proportion of ovulations that are detected. This has been called detection "efficiency." To estimate ovulation detection sensitivity, a sample of ovulations must be identified and the proportion of those ovulations that were detected by the system under evaluation calculated. Ovulations have been identified using progesterone concentrations in blood or milk samples collected from each cow every 2 to $7 \mathrm{~d}$ (Gümen et al., 2003), sometimes in combination with behavioral signs (de Mol and Woldt, 2001), examination of ovaries by rectal palpation (Peter and Bosu, 1986), or ultrasonic examination (Gümen et al., 2003). These methods are useful research tools but repeated collection of blood or milk samples and frequent examination of ovaries require substantial labor input, making these methods impractical for routine or even occasional use in many commercial dairy herds.

These practical limitations are overcome with alternative methods for assessing ODS that rely only on estrus and AI data, combined with pregnancy diagnosis. These indirect methods are based on intervals from calving to first postpartum estrus or AI, submission proportions, interestrual intervals, percentage of cows presented for pregnancy diagnosis that are not pregnant, and percentages of ovulations assumed that are detected (Gaines, 1989a). However, these methods have limitations. Some assess ODS over periods when efforts to detect ovulations may not be maximal. It is desirable to assess this specifically over a period of maximal effort; low sensitivity would indicate serious problems with herd reproductive management. A further limitation is that false positive AI can result in overestimation of ODS (Fetrow, 1993), and because PPV are quite variable between herds (Cavestany and Galina, 2001a), methods are required that account for these. Further, some methods fail to account for cows that have not initiated ovulatory cycles after calving can result in underestimation of ODS, even though the prevalence of anestrus just before the initiation of the breeding program can be high (McDougall, 2001; Gümen et al., 2003). Cows that have not begun postpartum ovulatory cycles could be accounted for by combining results of progesterone assays in strategically collected milk samples with estrus and AI data.

A large research project (the InCalf Project) was conducted in Australia from 1996 to 2000 (Morton, 2004). The project consisted of 2 prospective single cohort studies using both year-round and seasonal calving herds. Single cohort studies involve enrolling subjects before ascertaining the exposure status of each for risk factors of interest, and then assessing outcomes of interest during a specified follow-up period. The objectives of the research reported in this paper were 1) to design a practical method to assess ovulation detection performance in commercial dairy herds that uses limited numbers of strategically collected milk samples, assesses performance over the period when herd managers are making maximal effort to detect ovulations, and when assessing proportions of ovulations detected, accounts for false positive estrus diagnoses and for cows that have not recommenced postpartum ovulatory cycles; 2) to implement this method in a substantial number of year-round and seasonal calving commercial dairy herds; and 3) to describe the estimated ovulation detection performance in these herds.

\section{MATERIALS AND METHODS}

\section{Overview of Study}

A method for estimating herd ovulation detection performance was implemented in 43 year-round calving and 124 seasonal calving commercial dairy herds as part of a multi-centered, prospective single cohort study conducted at 11 sites located in the 4 eastern states of Australia (Morton, 2010). Strategically collected milk samples were assayed for progesterone. Assay results were combined with calving and AI data, and herdlevel estimates were calculated for PPV for ovulation diagnoses and ODS.

\section{Selection of Study Sites, Herds, and Lactations}

Eleven sites in 9 of Australia's 15 major dairy regions were purposefully selected to ensure a) that the majority of Australia's major dairy regions were represented, b) both year-round and Seasonal calving herds were enrolled, and c) a suitable site coordinator with access to sufficient numbers of herds was available at each site. Study sites were in Queensland (1 site each in Atherton Tablelands and southeast Queensland), New South Wales (1 site each in Camden and Bega), Victoria [Goulburn Valley (1 site), southwest Victoria (2 sites), 
south and east Gippsland (1 site each)], and northwest Tasmania (2 sites).

At each site, a private dairy veterinary practitioner acted as site coordinator. In total, they purposively selected 170 herds participating in milk production recording in which the herd manager would be likely to maintain accurate data records and complete study tasks as requested, where the herd manager was not planning to synchronize returns to estrus following AI and, for year-round calving herds, where the herd manager was not planning to run bulls continuously with the lactating herd. Herds with extremely unusual herd characteristics including atypical age structures (e.g., most cows in the herd being first-lactation cows) or purchasing and culling patterns not typical for commercial dairy herds (e.g., herds managed by cattle dealers) were ineligible. The expected herd size had to be $\geq 80$ cows in year-round calving herds and $\geq 120$ cows in seasonal calving herds. In addition, few herds with greater than 300 cows were selected.

In year-round calving herds (herds where cows calved in at least 10 calendar months each year), calvings within a 14- or 15-mo period from September or October 1996 to November 1997 were selected. For cows that calved twice in this period, the earliest lactation was selected. In seasonal calving herds (herds where approximately $90 \%$ of calvings each year occurred within a period of 150 consecutive days or less), a 12-mo period was used. For autumn- and winter-calving herds (most calvings occurred between March and August), this was from January to December 1997. For spring-calving herds (most calvings between August and October), this was from March 1997 to February 1998. Lactations in seasonal calving herds that commenced $>130 \mathrm{~d}$ before or $>59 \mathrm{~d}$ after the initiation of breeding program date were ineligible for analyses.

Reproductive performance was monitored in yearround calving herds to detect conceptions until at least $200 \mathrm{~d}$ after the initiation of the breeding program of the study lactation with the most recent calving date, and in seasonal calving herds until the end of the herd's breeding program, using insemination dates and results of subsequent pregnancy diagnoses.

\section{Determination of Voluntary Waiting Period and Initiation of Breeding Program Dates}

Voluntary waiting period was estimated for each year-round calving herd based on joint consideration of the distributions of intervals from calving to estrus without AI and intervals from calving to AI. The time from calving to when AI was first allowed was defined as the shortest time when intervals from calving to AI consistently predominated over intervals to estrus with- out AI; voluntary waiting period was calculated as $1 \mathrm{~d}$ less than this. Initiation of breeding program date for each cow was calculated as calving date plus the voluntary waiting period determined for that herd plus $1 \mathrm{~d}$. In each seasonal calving herd, the initiation of breeding program date was determined from distributions of AI by calendar date.

\section{Estimation of PPV for Ovulation Diagnoses}

Sampling Strategy. The sampling strategy was designed to result in collection of at least 50 milk samples from a representative selection of cows on the day that imminent ovulation was diagnosed by the herd manager or staff (Figure 1). This number was based on sample size calculations performed using WinPepi Version 8.6 (J. H. Abramson; http://www.brixtonhealth.com/); under simple random sampling, 48 samples were required from a population of 200 for a $95 \%$ CI of \pm 0.075 around a point estimate of 0.9 .

A regimen using simple random sampling of cows diagnosed in estrus was impractical and unlikely to be implemented correctly by herd managers and staff, so multistage sampling was used involving selecting cows from those diagnosed in estrus (first stage) on selected days (second stage). Dates were specified in advance for each herd by the site coordinator, and herd managers were asked to collect milk samples on those days from cows in which imminent ovulation had been diagnosed. Most samples were foremilk collected from a single quarter. Site coordinators selected dates after consideration of herd size and anticipated reproductive management so that at least 50 samples were collected. In year-round calving herds, milk samples were collected throughout the study. Sampling days were selected avoiding regular intervals of 7,14 , or $21 \mathrm{~d}$; this was done in case criteria for diagnosis of estrus varied between days during each week in a consistent pattern. In seasonal-calving herds, initially from the start of the breeding program, only AI was performed and bull services were not allowed; samples were collected throughout the AI period. In seasonal calving herds where the AI period was 6 wk or more, site coordinators were asked to specify sampling days such that 25 cows were sampled during the first 24 $\mathrm{d}$ and 25 were sampled throughout the remainder of the AI period. When fewer than 10 cows were diagnosed in estrus on a selected day, all were sampled. When 10 or more were diagnosed, cows were selected for milk sampling based on their identity number using preselected random numbers.

Calculation of Estimates. Progesterone assay results were matched with estrus and AI data. Diagnoses associated with high milk progesterone concentration $(\geq 5 \mathrm{ng} / \mathrm{mL}$ ) were considered false positive estrous di- 


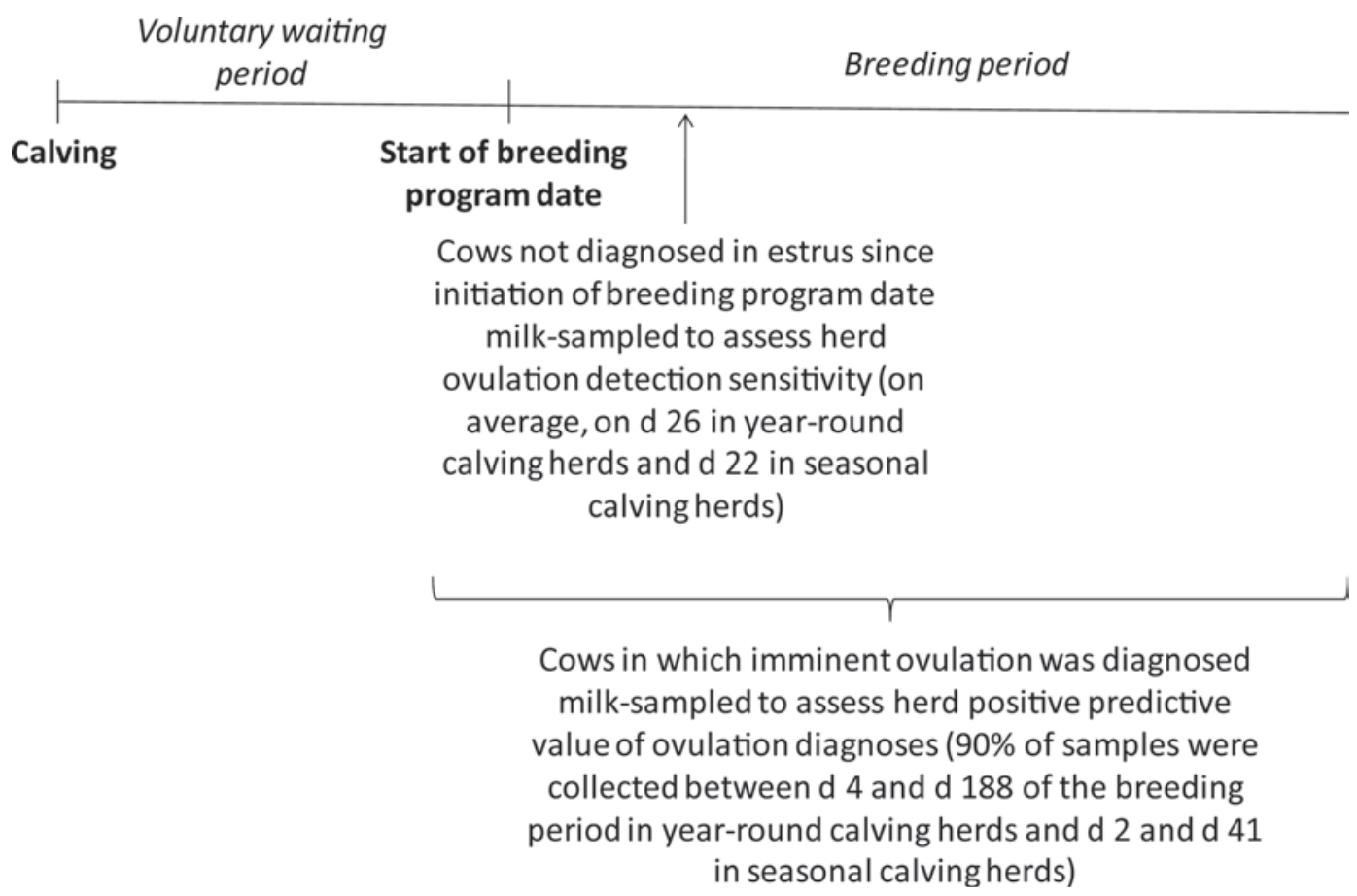

Figure 1. Overview of milk sampling strategy to estimate herd ovulation detection sensitivity and positive predictive value of ovulation diagnoses.

agnoses. Remaining cows were classified as about to ovulate or recently ovulated and were assumed correct diagnoses of ovulation for the purposes of this estimate. At the end of the study, PPV was calculated for each herd thus:

$$
1-\left(\frac{\text { No. of false-positive diagnoses }}{(\text { No. of false-positive diagnoses }+ \text { No. of correct diagnoses })}\right) .
$$

Herd managers were not informed of the estimated PPV or individual milk sample results until after the study.

\section{Estimation of ODS}

Overview. For a specified herd and time interval, ODS was defined as

$$
1-\left(\frac{\text { No. of undetected ovulations during interval }}{\text { No. of ovulations that occurred during interval }}\right),
$$

which equates to

$$
1-\left(\frac{\text { No. of undetected ovulations during interval }}{(\text { No. of undetected ovulations }+ \text { No. of detected ovulations })}\right) \text {. }
$$

Estimating Numbers of Undetected Ovulations. It was possible to estimate the number of undetected ovulations occurring during a specified time interval from each cow's initiation of breeding program date based on milk progesterone concentrations at the end of this interval in nonpregnant cows not diagnosed in estrus. Assuming that milk progesterone has very high diagnostic specificity and the prior probability of these cows being in the luteal phase was not zero, virtually all cows with high milk progesterone would be in the luteal phase when sampled. Assuming all of these cows had commenced normal cycling patterns by their initiation of breeding program date, on average, each would have ovulated once per $21 \mathrm{~d}$ from that date. This assumption of ovulations occurring every $21 \mathrm{~d}$ was supported by the distributions of intervals between estrus diagnoses in study herds, in which the highest peaks in the distributions were centered on $21 \mathrm{~d}$ in year-round calving herds, and 20 and $21 \mathrm{~d}$ in seasonal calving herds.

Sampled cows that had been diagnosed in estrus or AI between the initiation of the breeding program and date of sampling were excluded, as were sampled cows that, based on results of rectal pregnancy diagnoses, were considered pregnant at time of sampling to AI before their initiation of breeding program date.

In year-round calving herds, managers were asked to collect milk samples from cows not diagnosed in estrus between their breeding program initiation date and 24 
d later ("eligible" cows) on d 24 (Figure 1). To ensure that eligible cows were selected throughout the study, the first 2 cows becoming eligible for sampling in each calendar month were sampled and no further eligible cows were sampled during the remainder of the month. This restriction was relaxed during the study in some herds to increase the number of eligible cows that were sampled.

Cows eligible for sampling that had low milk progesterone concentrations $(<5 \mathrm{ng} / \mathrm{mL})$ when sampled were assumed not to have ovulated on or since their initiation of breeding program date and so contributed no ovulations to either the numerator or denominator in formula [3]. For eligible cows with high milk progesterone concentrations $(\geq 5 \mathrm{ng} / \mathrm{mL})$ when sampled, the likely number of ovulations from their initiation of breeding program date was estimated. Those cows with high milk progesterone concentrations that had not been treated with prostaglandin were assumed to have ovulated every $21 \mathrm{~d}$ from their initiation of breeding program date, so the likely number of ovulations was estimated for each of these cows as

$\underline{\text { Days from initiation of breeding program to sampling }}$ 21

Where cows with high milk progesterone concentrations had been treated with prostaglandin, the likely number of ovulations was estimated using a simulation model that incorporated response proportions and intervals to ovulation by day of estrous cycle when treated based on previous studies (Macmillan, 1983).

Within each year-round calving herd, cows were sampled at varying intervals after their initiation of breeding program date. The average of these intervals varied between herds from 9 to $43 \mathrm{~d}$. Cows that had not been diagnosed in estrus by their herd's average interval after their initiation of breeding program date but not sampled were assumed to have had the same number of undetected ovulations as sampled cows. The likely total number of undetected ovulations during the interval for the herd was calculated as the sum of the likely numbers of ovulations in the sampled cows divided by the proportion of eligible cows that were sampled.

In each seasonal calving herd, a specific date was selected for collection of milk samples from cows not diagnosed in estrus on or since the herd's initiation of breeding program date. In herds not using an estrous synchronization program, this selected collection date was approximately $24 \mathrm{~d}$ after the initiation of the herd breeding program date (Figure 1). When synchronization programs were used, earlier dates were selected (typically $14 \mathrm{~d}$ after the initiation of the herd breeding program date) to reduce the likelihood of cows with high progesterone having ovulated more than once between their initiation of breeding program date and sampling date. In 3 herds synchronizing 2 groups of cows, separate dates were selected for each group. Herd managers were requested to sample milk on the specified date(s) from 30 cows that had not been diagnosed in estrus on or since the initiation of breeding program date. In herds with more than 30 cows eligible for sampling, those with longest intervals since calving were sampled. Cows that had low milk progesterone concentrations $(<5 \mathrm{ng} / \mathrm{mL})$ when sampled were assumed not to have ovulated on or since their initiation of breeding program date and so contributed no ovulations to either the numerator or denominator in formula [3]. For cows with high milk progesterone concentrations $(\geq 5 \mathrm{ng} / \mathrm{mL})$, the likely number of ovulations on or since their initiation of breeding program date was estimated. Those cows with high milk progesterone concentration that had not been treated with prostaglandin were assumed to have ovulated every $21 \mathrm{~d}$ from the initiation of breeding program date so the likely number of ovulations was estimated for each of these cows using formula [4]. For cows with high milk progesterone concentration that had been treated with prostaglandin, the likely number of ovulations was estimated using a model that incorporated response proportions and intervals to ovulation by day of estrus cycle when treated based on previous studies (Macmillan, 1983) and distributions of intervals between ovulations (Larcombe, 1989). Some cows not diagnosed in estrus on or since the initiation of breeding program date were not sampled. These were assumed to have had the same number of undetected ovulations as sampled cows. The likely total number of undetected ovulations on or since the initiation of breeding program date for the herd was calculated as the sum of the likely numbers of ovulations in the sampled cows divided by the proportion of eligible cows that were sampled.

For seasonal calving herds, separate calculations were performed for early- and late-calved cows (calved $>6$ wk before the initiation of the breeding program or $\leq 6$ wk before, respectively) and then summed to provide a total for the herd. This stratified approach was used to minimize the potential for bias if different proportions of early- and late-calved cows not diagnosed in estrus were sampled. Relative to early-calved cows, a lower prevalence of undetected ovulation would occur among late-calved cows not diagnosed in estrus if the prevalence of anestrus was higher in this group as has been reported (McDougall, 2001). In the 3 herds that treated groups of cows with prostaglandins on 2 different days, separate calculations were performed for each group then summed. 
Estimating Numbers of Detected Ovulations. The total number of estrous periods and AI diagnosed in each herd to mean of times from each cow's initiation of breeding program date to sampling date (year-round calving herds) or from herd initiation of breeding program date until sampling date (seasonal calving herds) was ascertained from herd records. To adjust for false positive ovulation diagnoses among these, this number was multiplied by the herd PPV estimate.

Calculating $O D S$. Estimates of the total numbers of undetected and detected ovulations during the time interval were substituted into formula [3] and ODS estimates were calculated for each herd. Herd managers were not informed of the estimated herd ODS or individual milk sample results until the end of the study.

\section{Milk Sample Handling and Progesterone Assay}

Herd managers were supplied with $10-\mathrm{mL}$ polypropylene tubes with $2 \mathrm{mg}$ of bronopol (Harcros Chemicals, Rydalmere, Australia) and $20 \mu \mathrm{g}$ of methylene blue (HD Scientific Supplies, Wetherill Park, Australia) dried on the side of each tube. After collection, tubes were agitated, labeled with herd number, cow number, and sample collection date, frozen in domestic refrigerators, and transported on ice to the Dairy Research Unit at the University of Sydney at regular intervals. Stability studies conducted during the pilot study (results not shown) suggested that progesterone concentration was unaffected by this handling process. Samples were stored frozen at the Dairy Research Unit until assayed.

All samples were assayed at the Dairy Research Unit, The University of Sydney, as whole milk using a RIA. For most samples, laboratory staff were not aware of whether milk samples were collected for estimation of positive predictive value or ovulation detection sensitivity and had no access to details about the samples other than identifying data (i.e., herd and cow identity and date of collection). The assay procedure was modified from that described previously by Downing et al. (1995). The progesterone antiserum was raised in sheep by immunizing with 4-pregnen- $11 \alpha$-ol-3,20-dionehemisuccinyl-HSA. The cross reactivity of the antiserum with related steroids is shown in Table 1 . Tritiated progesterone $\left(1,2,6,7-{ }^{3} \mathrm{H}\right.$ progesterone: $\left.3.2 \mathrm{TBq} \cdot \mathrm{mmol}\right)$ was stored in ethanol at $-20^{\circ} \mathrm{C}$ and when required, the appropriate quantity was dried down with nitrogen gas and redissolved to $8,000 \mathrm{cpm}$ in $100 \mu \mathrm{L}$ of PBS buffer. Assay standards were diluted from a stock solution of $0.16 \mu \mathrm{g} / \mathrm{mL}$ in ethanol, dried under nitrogen gas, and redissolved in blank whole milk obtained from an ovariectomized cow to provide concentrations ranging from 0.1 to $12.8 \mathrm{ng} / \mathrm{mL}$ by serial dilution. A range of internal
Table 1. Cross reactivity of progesterone antiserum with related steroids in a RIA for progesterone used to assess ovulation detection performance in dairy herds

\begin{tabular}{|c|c|}
\hline Steroid & Cross reactivity, $\%$ \\
\hline 11ß-Hydroxy-progesterone & 17.5 \\
\hline Deoxycorticosterone & 9.5 \\
\hline $3 \beta$-Pregnene-3,20-dione & 2.5 \\
\hline 5 $\beta$-Pregnene-3,20-dione & 2.1 \\
\hline $17 \alpha$-Hydroxy-progesterone & $<0.1$ \\
\hline Cholesterol & $<0.1$ \\
\hline Cortisol & $<0.1$ \\
\hline Pregnenolone & $<0.1$ \\
\hline Testosterone & $<0.1$ \\
\hline Estradiol-17 $\beta$ & $<0.1$ \\
\hline
\end{tabular}

standards was prepared by adding standard solutions to blank plasma in known concentrations.

Whole milk samples $(100 \mu \mathrm{L})$ were diluted with 300 $\mu \mathrm{L}$ of PBS buffer and $2 \mathrm{~mL}$ of redistilled hexane. The tubes were capped and shaken for $10 \mathrm{~min}$ and the solvent and plasma phases separated by freezing the aqueous phase in an ethanol/dry ice bath and decanting the solvent phase. The extracted steroid was recovered from the solvent phase by heating on a dry block while evaporating with a stream of nitrogen and then dissolved in $100 \mu \mathrm{L}$ of PBS buffer. Samples were left for $30 \mathrm{~min}$ or overnight. The primary antiserum $(100 \mu \mathrm{L}$ of a working dilution of $1 / 6,000$ ) was added and left for $30 \mathrm{~min}$ at $23^{\circ} \mathrm{C}$ before adding the label. The assay was incubated at $4^{\circ} \mathrm{C}$ overnight. Dextran-coated charcoal $(0.25 \mathrm{~mL})$ was quickly added to each tube in defined batches (144 tubes per batch) and the contents vortexed and left for $15 \mathrm{~min}$ at $4^{\circ} \mathrm{C}$. The supernatant containing the antibody-bound radiolabel was decanted into a separate plastic vial. Scintillation cocktail $(3 \mathrm{~mL})$ was added and capped tubes were shaken and counted in a liquid scintillation counter (LKB-Wallac 1215, Wallac, Turku, Finland). The single phase counting gave an efficiency of around $30 \%$ while quenching was constant across the assay. Hormone concentrations were counted from standard curves using Multicalc software (LKBWallac). This generated a 4-parameter logistic spline smoothed standard curve. The sensitivity of the assay was $0.1 \mathrm{ng} / \mathrm{mL}$ and the intraassay coefficient of variation varied from $19.4 \%$ at $1.2 \mathrm{ng} / \mathrm{mL}$ to $16.1 \%$ at $2.6 \mathrm{ng} / \mathrm{mL}$ and $19.5 \%$ at $5.5 \mathrm{ng} / \mathrm{mL}(\mathrm{n}=65)$. The corresponding interassay coefficients of variation for the same concentrations were $18.5 \%, 13.3 \%$ and $16.9 \%$, respectively (n $=65$ ). Assay standard curves were most sensitive over the range of 0.5 to $10 \mathrm{ng} / \mathrm{mL}$. The recovery of progesterone added to whole milk from ovariectomized cows over this range of concentrations was $122.3 \pm 0.03 \%$ at $1 \mathrm{ng} / \mathrm{mL}(\mathrm{n}=65), 86.7 \pm 0.04 \%$ at $3 \mathrm{ng} / \mathrm{mL}(\mathrm{n}=33)$ and $107.4 \pm 1.9 \%$ at $5 \mathrm{ng} / \mathrm{mL}(\mathrm{n}=57)$. 
The receiver operating characteristics curve for the assay was developed using data from a pilot study involving 58 cows in 2 additional herds in which foremilk was sampled when cows were ovulating and again in the luteal phase, indicating that the assay was highly discriminatory between ovulating cows and those in the luteal phase (Morton, 2004). These seasonal calving herds had typical to high conception rates in previous mating periods (50 to $60 \%$, whereas the median from the herds in the current study was $49 \%$; Morton, 2004), indicating that the proportions of AI that were false positive estrous diagnoses in these 2 herds were probably low, and both used a whole-herd estrous synchronization program. Cows diagnosed in estrus by the herd manager on $\mathrm{d} 3$ after prostaglandin treatment that subsequently returned to estrus 19 to $25 \mathrm{~d}$ later or diagnosed by rectal examination 59 to $80 \mathrm{~d}$ later as having conceived to AI on d 3 were milk-sampled to provide ovulating samples. The same 58 cows were used to collect luteal phase samples; each cow was sampled again once between 4 and $16 \mathrm{~d}$ after they were originally sampled. A progesterone concentration threshold of 5 $\mathrm{ng} / \mathrm{mL}$ was used to classify each milk sample as either high or low (Morton, 2004). Sensitivity and specificity of progesterone concentration for detecting cows in the luteal phase were both greater than 0.85 for progesterone thresholds between approximately 5 and $11 \mathrm{ng} / \mathrm{mL}$, and specificity estimates were substantially less than 0.85 at lower thresholds. The threshold of $5 \mathrm{ng} / \mathrm{mL}$ was selected as this was the midpoint of the range over which the assay standard curves were most sensitive.

\section{Calculation of Interestrual Interval Ratios}

To allow comparison between ODS estimates and ratios of numbers of single (18 to $24 \mathrm{~d}$ ) to double (38 to $46 \mathrm{~d}$ ) interestrual intervals, a commonly used alternative estrus detection index (Heersche and Nebel, 1994) was used. The ratio was calculated for each year-round calving herd using all intervals and, separately, using only intervals commencing on or between $21 \mathrm{~d}$ before and $9 \mathrm{~d}$ after the cow's initiation of breeding program date. This ratio was not calculated for the seasonal calving herds because herd managers usually monitored the herd for estrus and recorded diagnoses only during the AI period, which was commonly only 4 to $8 \mathrm{wk}$ in duration (Morton, 2010). Thus, estrous detection in the first $18 \mathrm{~d}$ of the breeding program could not be assessed in most of these herds using this ratio as the ratio essentially assesses estrous detection sensitivity 18 to 24 $\mathrm{d}$ after the commencement of each interval. In addition, as only intervals commencing at least $46 \mathrm{~d}$ before the end of the AI period were used for these calculations, no or only small numbers of intervals were available to calculate ratios in most seasonal calving study herds.

\section{Statistical Analyses}

Correlations between PPV and ODS were assessed separately for year-round and seasonal calving herds with Spearman's rank correlation coefficients using Stata Version 10 (Stata Corp., College Station, TX). Associated 95\% confidence intervals were calculated using Fisher's transformation. Correlations between ODS and ratios of numbers of single to double interestrual intervals were assessed in the same way.

\section{RESULTS}

\section{Numbers of Herds}

Of the 170 herds selected for the study, 1.2\% (2/170) was lost to follow-up as the herd manager withdrew the herd from the project. In addition, 1 year-round-calving herd changed from AI of cows in estrus year-round to AI only within 4 distinct periods within each 12-mo period. Because this practice was not typical of reproductive management in year-round calving herds, ovulation detection performance statistics for this herd are not reported. The final study population for assessing ovulation detection performance consisted of 167 herds (43 year-round calving and 124 seasonal calving).

\section{PPV Estimates}

Milk samples were collected on day of AI in all 167 herds. The mean and median numbers of suitable samples collected per herd were 52 and 48 , respectively (range: 16 to 179). The estimated PPV from 1 yearround-calving herd was disregarded because the PPV estimate $(16 \%$ or $4 / 25)$ was extremely low and not consistent with the observed proportion of all AI in the herd that resulted in conception of $37 \%(191 / 519)$. Relative to other study herds, this herd had a disproportionately and inexplicably high proportion of samples with intermediate progesterone concentrations.

Descriptive statistics and distributions for PPV estimates for the remaining herds are in Table 2 and Figure 2. Although median estimates were high, PPV estimates varied widely between herds. Estimated PPV was $<0.95$ in $25 \%$ of herds. Distributions were leftskewed and were similar in year-round and seasonal calving herds.

\section{ODS Estimates}

Ovulation detection sensitivity could not be estimated in 5 of the 167 herds (3\%; all seasonal calving) because cows not diagnosed in estrus from the initiation of breeding program date to $24 \mathrm{~d}$ later did not have milk samples collected at the end of that interval. In the 


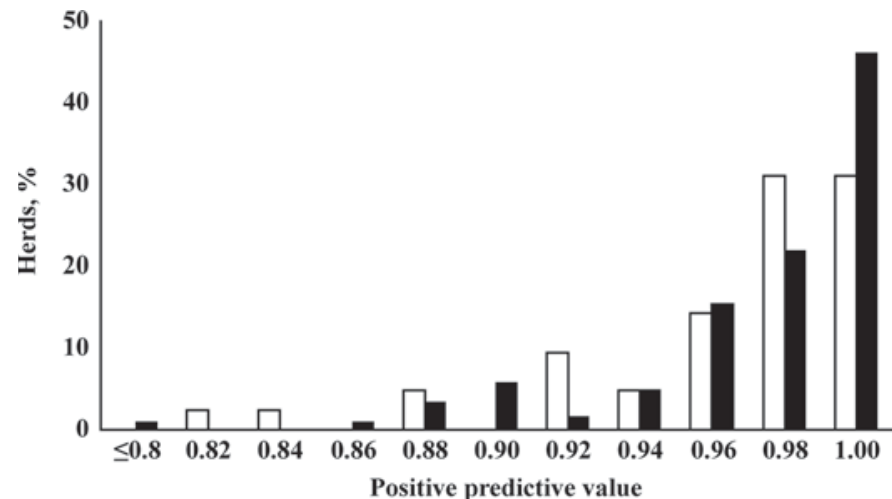

Figure 2. Distributions of positive predictive values (PPV) for 42 year-round calving (white bars) and 124 seasonal-calving (black bars) Australian dairy herds. A PPV category of 0.82 indicates values $>0.80$ and $\leq 0.82,0.84$ indicates values $>0.82$ and $\leq 0.84$, and so on.

remaining 162 herds, the mean and median numbers of cows not diagnosed in estrus on or since the initiation of breeding program date that were sampled per herd were 25 and 26, respectively (range: 1 to 98 ). On average, $60 \%$ of cows not diagnosed in estrus were sampled (median 65\%; range between herds: $1 \%$ to 100\%). For year-round and seasonal calving herds, the averages of the herd mean interval from the initiation of breeding program date to sampling date were $26 \mathrm{~d}$ and $22 \mathrm{~d}$, respectively.

Descriptive statistics and distributions for ODS estimates for the 162 herds are in Table 2 and Figure 3. Median estimated ODS was 0.73 in year-round calving herds and 0.94 in seasonal-calving herds. Within both calving systems, ODS estimates varied widely between herds. Estimated ODS was $<0.63$ in $25 \%$ of year-round calving herds and $<0.80$ in $25 \%$ of seasonal calving herds.

\section{Correlation Between ODS and PPV}

Within both year-round calving and seasonal calving herds, no important correlation was apparent between

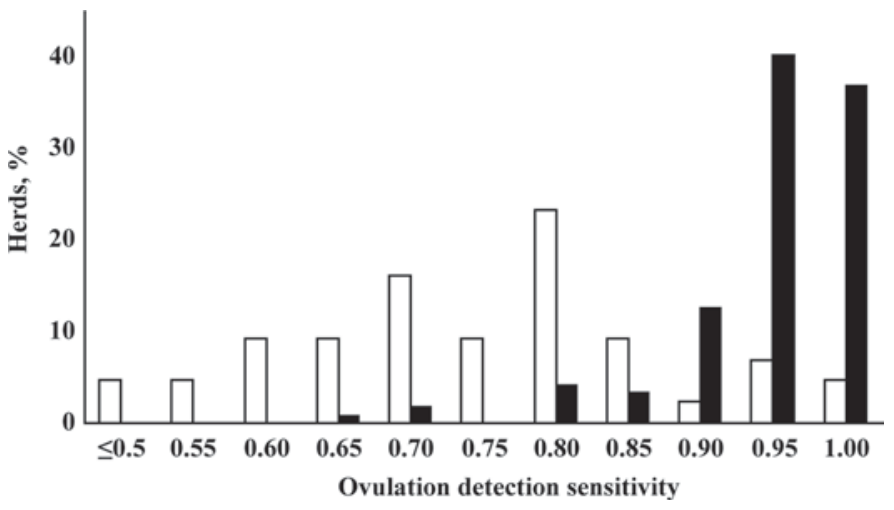

Figure 3. Distributions of ovulation detection sensitivity (ODS) for 43 year-round calving (white bars) and 119 seasonal-calving (black bars) Australian dairy herds. An ODS category of 0.55 indicates values $>0.50$ and $\leq 0.55,0.60$ indicates values $>0.55$ and $\leq 0.60$, and so on.

estimates for ODS and PPV. Spearman's rank correlation coefficients were both 0.00 (95\% CI: -0.30 to 0.31 ; $P=0.989$ and $95 \%$ CI: -0.18 to $0.18 ; P=0.996$ for year-round and seasonal calving herds, respectively).

\section{Correlations Between ODS and Single to Double Interestrual Interval Ratios}

The ODS estimates were not closely correlated with the ratio of numbers of single (18 to $24 \mathrm{~d}$ ) to double (38 to $46 \mathrm{~d}$ ) interestrual intervals whether using all intervals (Spearman's rank correlation coefficient 0.39; $95 \%$ CI: 0.10 to $0.61 ; P=0.011$ ) or only intervals commencing on or between $21 \mathrm{~d}$ before and $9 \mathrm{~d}$ after the cow's initiation of breeding program date (Spearman's rank correlation coefficient $0.22 ; 95 \%$ CI: -0.09 to 0.49 ; $P=0.154)$.

\section{DISCUSSION}

In this study, a method was developed to assess ovulation detection performance in commercial dairy herds that used limited numbers of strategically col-

Table 2. Descriptive statistics for positive predictive values (PPV) and ovulation detection sensitivity (ODS) for 43 year-round and 124 seasonal calving Australian dairy herds

\begin{tabular}{|c|c|c|c|c|c|c|}
\hline Item & Median & Mean & Minimum & 25th percentile & 75th percentile & Maximum \\
\hline \multicolumn{7}{|l|}{ PPV } \\
\hline Year-round calving herds $(\mathrm{n}=42)^{1}$ & 0.968 & 0.957 & 0.815 & 0.950 & 0.987 & 1.000 \\
\hline Year-round calving herds $(\mathrm{n}=43)$ & 0.727 & 0.723 & 0.458 & 0.634 & 0.797 & 1.000 \\
\hline Seasonal calving herds $(\mathrm{n}=119)^{2}$ & 0.938 & 0.923 & 0.637 & 0.911 & 0.961 & 1.000 \\
\hline
\end{tabular}

${ }^{1}$ The PPV estimate from 1 herd was excluded from analyses because of a disproportionately and inexplicably high proportion of samples with intermediate progesterone concentrations (see text for more details).

${ }^{2}$ ODS could not be estimated in 5 herds as none of the cows not diagnosed in estrus from the initiation of the breeding program to $24 \mathrm{~d}$ after that date were milk sampled at the end of that interval. 
lected milk samples, assesses performance over the period when herd managers are making maximal effort to detect ovulations, and when assessing proportions of ovulations detected, accounts for false positive estrus diagnoses and for cows that have not recommenced postpartum ovulatory cycles. The method was successfully implemented in the majority of commercial dairy herds selected for this study. In a minority of herds, implementation was only partly successful. In 5 of the 167 herds, no samples were collected from any of the cows eligible to be sampled to estimate ODS, and in 1 herd, PPV could not be estimated because an inexplicably high proportion of samples had intermediate progesterone concentrations. These observations indicate that this system can be successfully implemented in many commercial dairy herds, but that the milk sampling requirements may be onerous for some herd managers. Veterinarians planned the sampling strategy for each herd with the herd manager and this support was probably essential for successful implementation in most herds.

Estimated PPV in study herds were high. Estimates in previous studies vary considerably with similarly high estimates in some studies (Royal et al., 2000), but much lower estimates in others (Sturman et al., 2000; Cavestany and Galina, 2001a; Gümen et al., 2003). Some variation in estimates between studies may be caused by differences in progesterone concentration thresholds. In the current study, the threshold of $5 \mathrm{ng} /$ $\mathrm{mL}$ was chosen based on estimates of sensitivity and specificity of progesterone concentration in foremilk for detecting cows in the luteal phase from a pilot study involving 58 cows sampled when ovulating and again when in the luteal phase.

In the current study, PPV were probably overestimated because AI of cows 2 to several days before ovulation and after ovulation and those with anovulatory anestrus would have been associated with low milk progesterone even though these were false positive AI. The extent of overestimation for these reasons was probably minimal in most herds. If progesterone concentration among cycling cows were above the selected threshold on only $64 \%$ of days when ovulation was not imminent (Lamming and Darwash, 1998), a PPV of 0.97 (the median for year-round calving herds) estimated using methods in the current study would reflect a true PPV of 0.95 \{calculated as $1-[(1-0.97) / 0.64]\}$, indicating minimal overestimation. Important overestimation would occur using methods from the current study for this reason only at much lower PPV. The extent of overestimation due to AI among anestrus cows was probably minimal in most herds. High proportions of cows may not have commenced cycling by $60 \mathrm{~d}$ postcalving (Gümen et al., 2003) and the prevalence of anestrus just be- fore the herd initiation of breeding program date in seasonal calving herds can be high. However, anestrus prevalence probably declined rapidly after the breeding program initiation dates in most study herds because the proportion of cows that have not commenced cycling decreases rapidly with time from calving (Jonsson et al., 1999). Further, even if the anestrus prevalence throughout the AI period were $30 \%$, a PPV of 0.97 based on methods in the current study would reflect a true PPV of 0.96 \{calculated as $1-[(1-0.97) /$ $(1-0.30)]\}$, indicating minimal overestimation. If the risk of false positive AI were less in anestrus cows than in cycling cows where ovulation was not imminent, the extent of this overestimation would be smaller.

The high PPV observed in this study suggest that diagnoses of estrus had extremely high specificity. Specificity in this context can be defined as the proportion of non-ovulating days where no diagnosis of estrus was made. For a given ODS, PPV increases with both specificity of diagnoses of estrus and the prevalence of ovulation on any particular day. In dairy herds, the prevalence of ovulation on any particular day is usually quite low. In the absence of estrous synchronization, the average prevalence among cows from the initiation of their breeding program to positive pregnancy diagnosis would be $<4.8 \%$. At these low prevalences, to achieve a PPV of, for example, 0.968 (the median estimate for year-round calving herds in the current study), specificity must be almost $100 \%$, indicating that estrus was rarely diagnosed on nonovulating days in many herds.

In $25 \%$ of study herds, estimates were $<0.95$. These low PPV likely resulted in reduced conception rates because probability of conception was lower following AI when progesterone concentrations were high (Gowan et al., 1982; Sturman et al., 2000), and false positive AI can reduce probability of conception to previous AI through disrupting existing pregnancies (Sturman et al., 2000). There is a need to assess effects of these low PPV on primary measures of herd reproductive performance such as proportion pregnant by wk 6 after the initiation of the breeding program (Morton, 2004).

Ovulation detection sensitivities may have been overestimated in the current study where estimates were low, such as in most year-round calving study herds. The method was based on the assumption that all sampled cows with low progesterone when sampled had not ovulated since the initiation of their breeding program. As some of the sampled cows not diagnosed in estrus that had low milk progesterone concentration would have been cycling, numbers of undetected ovulations in each herd would have been underestimated. The likely extent of bias in ODS estimates caused by these cows can be estimated. If progesterone concentration among cycling cows were above the selected threshold on only $64 \%$ of 
days when ovulation was not imminent (Lamming and Darwash, 1998), an ODS estimate of 0.73 would reflect a true ODS of 0.63 , indicating important overestimation. In contrast, the extent of overestimation would have been much less with higher ODS estimates. For example, an ODS estimate of 0.94 would reflect a true ODS of 0.91, indicating minimal overestimation. Cows having interovulatory intervals of $<21 \mathrm{~d}$ would result in overestimation if all of their ovulations between their initiation of breeding program date and milk sampling date were undetected. This overestimation was probably partly counterbalanced by some cows having intervals longer than $21 \mathrm{~d}$ between ovulations (Lamming and Darwash, 1998; Gümen et al., 2003). Variation in interovulatory intervals would not be a source of bias if the average of intervals between initiation of the breeding program and milk sampling were $21 \mathrm{~d}$. Finally, the current method assessed ODS over the first 1 to $4 \mathrm{wk}$ after initiation of breeding program dates. If this declined during each cow's breeding program or after their first AI, then the current method would overestimate overall ODS. Because of these sources of overestimation, low ODS using the current method can be viewed as a highly specific indicator of low estrous detection. In herds where ODS using the current method appears adequate, further assessments of estrous detection may be indicated.

The generally high ODS estimates from seasonal calving herds were consistent with previous work (Gill, 1985). Despite this, there was large variation between herds in the current and previous (Gill, 1985) studies, indicating that there was potential for substantial increases in ODS in some Australian seasonal calving herds. This potential may be greater than indicated because ODS was estimated for the early part of the breeding program and this was the period of the intense management effort to detect estrus. The estimates of estrous detection performance from the study herds may be biased upward relative to Australian herds, because it was not practical to select study herds randomly. To maximize data quality, our selection was biased toward herds in which the herd manager was likely to maintain accurate data records and complete study tasks as requested. It is likely that data quality would have been reduced in a randomly selected group of herds. Overestimation would have occurred if these types of managers had better estrous detection performance. Overestimation may have occurred if herd managers and staff improved estrous detection because they were involved in the study, a phenomenon referred to as the "Hawthorne effect" (Wickstrom and Bendix, 2000).

The current study supported previous studies showing that ODS was low in year-round calving herds (Shipka,
2000; Cavestany and Galina, 2001b; Delwiche et al., 2001). The higher ovulation detection sensitivities in seasonal calving herds relative to year-round calving herds may be due to both the relatively short periods where AI is used (Morton, 2004) and higher prevalences of ovulation in seasonal calving herds. Shorter periods of estrous detection facilitate more intense efforts to detect estrus than are possible when year-round estrous detection is necessary, and high numbers of cows in estrus simultaneously (as is common in seasonal calving herds) may also increase chance of detection as both number of mounts per ovulatory period (Hurnik et al., 1975 ) and duration of estrus behaviour (Van Vliet and Van Eerdenburg, 1996) are increased if 2 or 3 cows are in estrus simultaneously.

The lack of strong association between ODS and PPV indicated that high ODS could be achieved without substantially compromising PPV. Several researchers suggested that false positive AI frequency increases when herd managers and staff attempt to increase estrous detection probability (Gaines, 1989b; Fetrow, 1993), because they inseminate cows showing signs less predictive of estrus. Estrous signs associated with an increased risk of false positive diagnoses have been reported (Reimers et al., 1985). However, herd managers inseminate a high proportion of cows not seen standing to be mounted but showing other signs, and moderate proportions of these AI were successful (Reimers et al., 1985). This suggests that a substantial proportion of cows not seen standing to be mounted were in estrus, and failure to AI would have reduced ODS. It is likely that managers of herds achieving both very high ODS and PPV optimized their criteria for selection of cows for AI to achieve very high herd reproductive performance.

Estimates of ODS were not correlated with the ratio of numbers of single (18 to $24 \mathrm{~d}$ ) to double (38 to 46 d) interestrual intervals. This may be because of different periods in which ovulation detection performance was assessed. Whereas ODS estimates from the current study assessed performance during the first 1 to 4 wk of the breeding program, the ratio assessed performance over a wider period. To partly address this, ratios were also calculated using only intervals commencing soon before or early in each cow's initiation of breeding program date. But these were not closely correlated with ODS, possibly because only small numbers of intervals were available for calculating these ratios, and estimated ratios for each herd were imprecise. Further research is required to compare various measures of ovulation detection performance in commercial dairy herds, and to assess the accuracy and cost-effectiveness of various methods. 


\section{CONCLUSIONS}

These findings indicate that this method for assessing ovulation detection performance can be successfully implemented in commercial dairy herds with appropriate professional support. The wide range of ovulation detection sensitivities and absence of correlation between ODS and PPV suggest that it is possible for managers of many commercial herds in Australia to achieve increased reproductive efficiency through increases in both ODS and PPV.

\section{ACKNOWLEDGMENTS}

It is a pleasure to acknowledge the Dairy Research and Development Corporation (now Dairy Australia) who funded the InCalf Project; the Project Management Committee (Mike Larcombe, Maffra Herd Improvement Co-operative, Maffra, Victoria; Pauline Brightling, The University of Melbourne, Werribee, Victoria; John Craven, Dairy Research and Development Corporation, Melbourne, Victoria; Chris Hibburt, Timboon Veterinary Group, Timboon, Victoria; Ian Lean, The University of Sydney and Bovine Strategic Services, Camden, New South Wales; Jock Macmillan, The University of Melbourne, Werribee, Victoria; Tony Martin, Agriculture WA, Bunbury, Western Australia; Michael McGowan, The University of Queensland, Brisbane, Queensland; Greg Stevens, Primary Industries South Australia, Flaxley, South Australia; and Bill Tranter, Tableland Veterinary Service, Malanda, Queensland); the site coordinators (Bill Tranter; Michael McGowan; Ian Lean; the late Bruce Adams, Bega Veterinary Clinic, Bega, New South Wales; Rod Dyson, Kyabram Veterinary Clinic, Kyabram, Victoria; Jakob Malmo, Maffra Veterinary Centre, Maffra, Victoria; Michael Pyman, Korumburra Veterinary Clinic, Korumburra, Victoria; Dave Colson, Allansford and Wollaston Veterinary Clinic, Warrnambool, Victoria; Peter Younis, Timboon Veterinary Group, Timboon, Victoria; Graeme Stephensen, Smithton Veterinary Clinic, Smithton, Tasmania; and Graham Harrison, Wynyard Veterinary Clinic, Wynyard, Tasmania) and many other veterinarians from their practices; Jo-Anne Sclater from the Dairy Research Unit at the University of Sydney; the 168 farming families whose herds were studied; Ivan Caple, The University of Melbourne, Werribee, Victoria (supervisor of John Morton's PhD); Dave Beggs, Warrnambool Veterinary Clinic, Warrnambool, Victoria; Mark Stevenson, EpiCentre, Institute of Veterinary, Animal \& Biomedical Sciences, Massey University, Palmerston North, New Zealand; and Norm Williamson, CHAMP Informatics Limited, Palmerston North, New Zealand, for making modifications to the three commercial software packages used during the project.

\section{REFERENCES}

Cavestany, D., and C. S. Galina. 2001a. Evaluation of an artificial insemination programme in a seasonal breeding dairy system through milk progesterone. Reprod. Domest. Anim. 36:79-84.

Cavestany, D., and C. S. Galina. 2001b. Factors affecting the reproductive efficiency of artificial insemination programmes in a seasonal breeding pasture-based dairy system with the aid of milk progesterone. Reprod. Domest. Anim. 36:85-89.

de Mol, R. M., and W. E. Woldt. 2001. Application of fuzzy logic in automated cow status monitoring. J. Dairy Sci. 84:400-410.

Delwiche, M., X. Tang, R. BonDurant, and C. Munro. 2001. Estrus detection with a progesterone biosensor. Trans. ASAE 44:20032008.

Downing, J. A., J. Joss, P. Connell, and R. J. Scaramuzzi. 1995. Ovulation rate and the concentrations of gonadotrophic and metabolic hormones in ewes fed lupin grain. J. Reprod. Fertil. 103:137-145.

Fetrow, J. 1993. Herd health monitoring and records analysis. Bovine Pract. 27:88-101.

Gaines, J. D. 1989a. The role of record analysis in evaluating subfertile dairy herds. Vet. Med. 84:532-543.

Gaines, J. D. 1989b. Working up the subfertile dairy herd: Assessing estrus detection and semen handling. Vet. Med. 84:636-644.

Gaines, J. D., C. B. Thomas, and S. Eicker. 1993. The interoestrous interval profile of a dairy herd: How useful is it? Vet. Med. 88:665-671.

Geers, R., B. Puers, V. Goedseels, and P. Wouters. 1997. Electronic identification, monitoring and tracking of animals. Cab International, Wallingford, UK.

Gill, I. J. 1985. Parameters and targets for dairy herd fertility. The challenge: Efficient dairy production. Pages $360-361$ in Proceedings of the conference organized by the Australian and New Zealand Societies of Animal Production., Albury Wodonga, Australia.

Gowan, E. W., R. J. Etches, C. Bryden, and G. J. King. 1982. Factors affecting accuracy of pregnancy diagnosis in cattle. J. Dairy Sci. 65:1294-1302.

Gümen, A., J. N. Guenther, and M. C. Wiltbank. 2003. Follicular size and response to Ovsynch versus detection of estrus in anovular and ovular lactating dairy cows. J. Dairy Sci. 86:3184-3194.

Heersche, G. Jr., and R. L. Nebel. 1994. Measuring efficiency and accuracy of detection of estrus. J. Dairy Sci. 77:2754-2761.

Hurnik, J. F., G. J. King, and H. A. Robertson. 1975. Estrous and related behaviour in postpartum Holstein cows. Appl. Anim. Ethol. 2:55-68.

Jonsson, N. N., W. J. Fulkerson, P. M. Pepper, and M. R. McGowan. 1999. Effect of genetic merit and concentrate feeding on reproduction of grazing dairy cows in a subtropical environment. J. Dairy Sci. 82:2756-2765.

Lamming, G. E., and A. O. Darwash. 1998. The use of milk progesterone profiles to characterise components of subfertility in milked dairy cows. Anim. Reprod. Sci. 52:175-190.

Larcombe, M. T. 1989. The effects of manipulating reproduction on the productivity and profitability of dairy herds which graze pasture. $\mathrm{PhD}$ thesis. University of Melbourne, Melbourne, Australia.

Macmillan, K. L. 1983. Prostaglandin responses in dairy herd breeding programmes. N. Z. Vet. J. 31:110-113.

McDougall, S. 2001. Effects of periparturient diseases and conditions on the reproductive performance of New Zealand dairy cows. N. Z. Vet. J. $49: 60-67$.

Meena, R. S., S. S. Sharma, and G. N. Purohit. 2003. Efficiency of vaginal electrical resistance measurements for oestrous detection and insemination in Rathi cows. Anim. Sci. 76:433-437.

Morton, J. 2004. Determinants of reproductive performance of dairy cows in commercial herds in Australia. PhD thesis. University of Melbourne, Melbourne. http://repository.unimelb.edu. au/10187/1503 Accessed Feb. 1, 2010. 
Morton, J. M. 2010. Interrelationships between herd-level reproductive performance measures based on intervals from initiation of the breeding program in year-round and seasonal calving dairy herds. J. Dairy Sci. 93:901-910.

Peter, A. T., and W. T. K. Bosu. 1986. Postpartum ovarian activity in dairy cows: correlation between behavioral estrus, pedometer measurements and ovulations. Theriogenology 26:111-115.

Reimers, T. J., R. D. Smith, and S. K. Newman. 1985. Management factors affecting reproductive performance of dairy cows in the Northeastern United States. J. Dairy Sci. 68:963-972.

Royal, M. D., A. O. Darwash, A. P. F. Flint, R. Webb, J. A Woolliams, and G. E. Lamming. 2000. Declining fertility in dairy cattle: changes in traditional and endocrine parameters of fertility. Anim. Sci. 70:487-501.
Shipka, M. P. 2000. A note on silent ovulation identified by using radiotelemetry for estrous detection. Appl. Anim. Behav. Sci. 66:153-159.

Sturman, H., E. A. B. Oltenacu, and R. H. Foote. 2000. Importance of inseminating only cows in estrus. Theriogenology 53:1657-1667.

Van Vliet, J. H., and F. J. C. M. Van Eerdenburg. 1996. Sexual activities and oestrus detection in lactating Holstein cows. Appl. Anim. Behav. Sci. 50:57-69.

Wickstrom, G., and T. Bendix. 2000. The "Hawthorne effect"-What did the original Hawthorne studies actually show? Scand. J. Work Environ. Health 26:363-367. 